\title{
Validation of methods for converting the original Disease Activity Score (DAS) to the DAS28
}

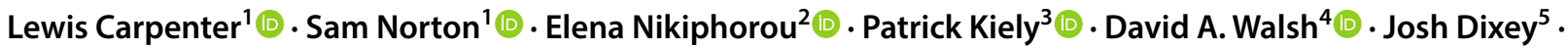 \\ Adam Young ${ }^{6}$ (1)
}

Received: 10 October 2018 / Accepted: 20 October 2018 / Published online: 27 October 2018

(c) The Author(s) 2018

\begin{abstract}
The Disease Activity Score (DAS) is integral in tailoring the clinical management of rheumatoid arthritis (RA) patients and is an important measure in clinical research. Different versions have been developed over the years to improve reliability and ease of use. Combining the original DAS and the newer DAS28 data in both contemporary and historical studies is important for both primary and secondary data analyses. As such, a methodologically robust means of converting the old DAS to the new DAS28 measure would be invaluable. Using data from The Early RA Study (ERAS), a sub-sample of patients with both DAS and DAS28 data were used to develop new regression imputation formulas using the total DAS score (univariate), and using the separate components of the DAS score (multivariate). DAS were transformed to DAS28 using an existing formula quoted in the literature, and the newly developed formulas. Bland and Altman plots were used to compare the transformed DAS with the recorded DAS28 to ascertain levels of agreement. The current transformation formula tended to overestimate the true DAS28 score, particularly at the higher end of the scale. A formula which uses all separate components of the DAS was found to estimate the scores with a higher level of precision. A new formula is proposed that can be used by other early RA cohorts to convert the original DAS to DAS28.
\end{abstract}

Keywords Rheumatoid arthritis · Epidemiology · Observational studies $\cdot$ Statistical methods

Electronic supplementary material The online version of this article (https://doi.org/10.1007/s00296-018-4184-0) contains supplementary material, which is available to authorized users.

Lewis Carpenter

lewis.carpenter@kcl.ac.uk

Sam Norton

sam.norton@kcl.ac.uk

Elena Nikiphorou

enikiphorou@gmail.com

Patrick Kiely

p.kiely500@btinternet.com

David A. Walsh

David.Walsh@nottingham.ac.uk

Josh Dixey

josh.dixey@nhs.net

Adam Young

adam.young@nhs.net

\section{Introduction}

The Disease Activity Score (DAS) is a composite score developed as a means of quantifying the severity of rheumatoid arthritis (RA) [1]. It is central in the current 'treat-to-target' (T2T) paradigm [2]. It is the most widely

1 Health Psychology Section, King's College London, London, UK

2 Department of Rheumatology, King's College London, London, UK

3 Department of Rheumatology, St George's University Hospital NHS Foundation Trust, London, UK

4 Arthritis UK Pain Centre, University of Nottingham, Nottingham, UK

5 Department of Rheumatology, Wolverhampton NHS Trust, Wolverhampton, UK

6 Postgraduate Medicine, University of Hertfordshire, Hatfield, UK 
used 'tool' in clinical practice to assess and monitor the patients' disease activity status and to help guide treatment adjustments. In the UK, the DAS28 is one of the main determinants in deciding access to biologic diseasemodifying antirheumatic drugs (bDMARDs) [3].

Several versions of the DAS exist. The original DAS established that four measures could be combined to give an effective overview of the overall disease status of a patient: a 44 joint Swollen Joint Count (SJC), the Ritchie Articular Index (RAI) of 53 tender joints, the Erythrocyte Sedimentation Rate (ESR) and a Patient Global Assessment (PGA) of disease activity on a $100 \mathrm{~mm}$ visual analogue scale (VAS) [1]. A three-variable calculation excluding the PGA is also available. In 1995, a modified version of the DAS was proposed, the DAS28, which used counts of 28 joints for both the SJC and tender joint count (TJC) focusing on the hands and arms [4]. Discrepancies between the two DAS scoring methods has been shown to occur, in part, by the omission of the feet and ankles in the DAS28 joint count [5]. However, the DAS28 is able to discriminate between patients with varying levels of disease activity and was preferred due to its simplicity and feasibility in the clinical setting. Other variations of the DAS and DAS28 exist, including the use of C-reactive protein (CRP) in place of ESR. However, the original four-variable DAS and DAS28 using ESR remain the most routinely used methods [6], and are part of the core set of outcomes in RA clinical trials [7].

It is agreed that the DAS and DAS28 scores cannot be used inter-changeably due to their differences in minimum and maximum values, and weighting used for each component of the score [6]. However, comparing these data would provide the opportunity to study longitudinal cohorts where different DAS methods have been used (e.g., Norfolk Arthritis Register (NOAR) [8] and the Yorkshire Early Arthritis Register (YEAR) [9], along with many other registries worldwide [10]), and to enable meta-analyses across studies reporting different DAS outcomes. This was the case in early data collected from The Early RA Study (ERAS), which collected the three-variable DAS for patients diagnosed between 1986 and 2001, while the Early RA Network (ERAN) collected DAS28 data for patients diagnosed between 2002 and 2011 [11].

Methods for transforming the original DAS to the DAS28 metric have been proposed, and used in a number of previous studies to try and facilitate direct comparisons [11-14]. A commonly used formula was devised by van Gestel et al. in 1998 using longitudinal data from an observational cohort [15]. Although precise details about how the formula was derived are not shown, it is likely to represent a regression imputation of the original DAS regressed on DAS28 scores to impute the missing DAS28 scores. While DAS and DAS28 scores are known to correlate highly, there is currently no research to assess the reliability and validity of this imputation method.

Using a sub-group of patients from the ERAS cohort attending one centre where both DAS and DAS28 data are available, this paper explores the use of imputation methods, including the formula given by van Gestel et al., to calculate missing DAS28 scores where DAS are recorded. The analyses will compare levels of agreement of (1) the van Gestel formula, (2) univariable regression imputation using DAS to predict DAS28, and (3) multivariable regression imputation model using the separate components of DAS to predict DAS28. Finally, the paper will examine differences in trends over time using the different methods to calculate DAS28 to determine potential differences in interpretation. The aim is to provide a potentially more statistically reliable transformation formula for converting DAS to DAS28.

\section{Methods}

\section{Data}

The Early RA Study (ERAS) recruited a total of 1465 patients with early RA from nine centres in England between 1986 and 2001. All patients had a confirmed diagnosis of RA and were recruited within 2 years of symptom onset (median 6-months), prior to conventional DMARD initiation. All patients were treated based on standard clinical practice of the time. Standard clinical, laboratory and radiographic data were collected during outpatient appointments at baseline, 6 months, 12 months, and then yearly thereafter. Each centre closed to follow up at different times between 2001 and 2013 with a maximum follow-up of 30 years (median 10 years) for annual outpatient follow-up. ERAS received retrospective ethical approval from the East Hertfordshire Local Research Ethics Committee in 1994 and subsequently from the Caldicott Guardian.

The analysis uses a subset of the main ERAS dataset with data from one centre $(n=298)$, where the component variables for both the DAS and DAS28 were recorded for the same patients by the same examiner. Restricting the analyses to the first 5 years, the 298 patients contributed a total of 1470 observations (mean 4.9 observations per patient).

\section{Disease activity scores}

All patients in ERAS had the 44-count SJC, the RAI, and ESR collected at each visit. The PGA was not routinely collected by centres, so the three-variable DAS formula was used to calculate the original DAS for all patients [1] (Eq. 1).

$$
\begin{aligned}
\operatorname{DAS}(3)= & (0.54 \times \operatorname{sqrt}(\mathrm{RAI}))+0.065 \times(\mathrm{SJC} 44) \\
& +0.33 \times \ln (\mathrm{ESR})+0.22 .
\end{aligned}
$$


For the subset of patients where the DAS28 component variables were collected, DAS28 was calculated using the four-variable formula [4] (Eq. 2)

$$
\begin{aligned}
\operatorname{DAS28}(3)= & (0.56 \times \operatorname{sqrt}(\mathrm{TJC} 28))+(0.28 \times \operatorname{sqrt}(\mathrm{SJC} 28)) \\
& +0.70 \ln (\mathrm{ESR}) \times 1.07+0.14 \times \text { PGA. }
\end{aligned}
$$

While a PGA VAS was not routinely collected, patient assessment of global pain using a $0-100$ VAS was. PGA was used where available but where missing (94\% observations) the pain VAS was used in place. The large proportion of missing PGA data was due to the fact that PGA data was typically collected beyond the 5-year follow-up for patients recruited from this centre. Khan et al. indicates that different measures of global health can be used inter-changeably without significant impact on the DAS [16]. This is likely due to the very high correlation between PGA and other measures of patient global health (i.e., PGA with pain VAS correlation was $r=.900$ in the current data). Sub-analysis (not shown) found that there was higher agreement between the DAS28 and DAS28 using pain VAS compared to just the three-variable DAS28 score, therefore the four-variable DAS28 with either the PGA or pain VAS was used.

The conversion formula given by van Gestel et al. [15] for imputing DAS28 based on DAS is shown in Eq. (3). The intention of the formula is to convert the DAS score to the DAS28 metric, however, it should be noted that essentially, as the multiplication factor is close to one, the formula simply increases the DAS score by 0.9 units.

DAS28 $=((1.072) \times$ DAS $)+0.938$

\section{Analysis}

The analysis examines three methods of imputing DAS28. Whilst other imputation methods have proven more effective in imputing missing data, such as multiple imputation [17], this is typically not possible in the context of transforming DAS to DAS28, as no data is available for the DAS28. As such, this paper focuses only on the use of regression imputation methods to devise a formula for wider use.

Univariable mixed-effects linear regression models regressed the DAS28 on to the DAS for the DAS-only regression imputation. Mixed models were used to account for the longitudinal nature of the data. The fixed effects from the model, the coefficient for DAS and the constant, were then used to calculate imputed DAS28 scores. A similar procedure was applied for the multivariable mixedeffects linear regression imputation using the individual DAS components: the square-root of the 44-count SJC and the RAI, the natural logarithm of ESR and PGA/pain VAS scores will be used. Additionally, sex (coded male 1 and female 2) and age in years were also included as predictors, since these are known to independently impact on
ESR [18]. The coefficient of each predictor, along with the constant, was then used to calculate imputed DAS28 scores. To evaluate the performance of the models, the model fit statistics were examined.

Mean scores of the DAS28 over the first 5 years will be compared to the three imputation methods outlined, and the mean difference, along with levels of agreement, will be assessed using Bland and Altman plots [19]. Bland and Altman plots are an effective way of assessing agreement between two scores and plots the difference between the two scores against the average of both scores. The Bland and Altman plots also provide summary statistics on the agreement between the scores using the mean difference, as well as providing $95 \%$ limits of agreement ( $\mathrm{LoA}$ ) around this mean estimate. Much like a $95 \%$ confidence interval, these limits are approximately 2 standard deviations above and below the mean difference. The closer the mean difference is to zero, the higher the agreement. The narrower the 95\% LoA, the less variation there is around this mean estimate [19]. The intra-class correlation coefficient (ICC) will also be used as another measure of agreement between each imputation method and the actual DAS28 scores. Finally, the DAS2 8 will be categorised into remission, low disease activity (LDA), moderate disease activity (MDA) and high disease activity (HDA) according to the EULAR DAS28 thresholds [20]. The proportion of patients categorised into each group will be assessed graphically, and agreement across categories will be assessed using the Kappa statistic [21].

\section{Results}

\section{Demographic data of patient sub-sample}

Of the patients from ERAS ( $n=1465), 298$ (20.3\%) attending one centre had the component variables for both DAS and DAS28 collected. The 298 patients contributed 1470 observations over 5 years (mean 4.9 observations per patient). Demographic and baseline clinical measures for the whole cohort, and those with and without DAS28 measures over the first 5 years are shown in Table 1. Patients with both DAS and DAS28 data were slightly older, more likely to be female and less likely to be seropositive. Baseline HAQ levels were similar across the groups, however, patients with recorded DAS28 indicated higher levels of baseline DAS scores when compared to the rest of the cohort. This is reflected by increased SJC, RAI and pain VAS scores at baseline, although baseline ESR was marginally lower. 
Table 1 Descriptive Statistics of ERAS cohort with and without DAS data

\begin{tabular}{|c|c|c|c|}
\hline & Total & ERAS (DAS only) & $\begin{array}{l}\text { ERAS (DAS and } \\
\text { DAS28) }\end{array}$ \\
\hline & $N=1465$ & $N=1167$ & $N=298$ \\
\hline \multicolumn{4}{|l|}{ Age at onset (years) } \\
\hline Mean (standard deviation) & $55.3(14.57)$ & $54.62(14.36)$ & $57.97(15.09)$ \\
\hline Female, $n(\%)$ & $973(66.42 \%)$ & $769(65.90 \%)$ & $204(68.46 \%)$ \\
\hline \multicolumn{4}{|l|}{ Baseline DAS } \\
\hline Mean (standard deviation) & $4.23(1.63)$ & $4.07(1.51)$ & $4.85(1.91)$ \\
\hline Median (inter-quartile range) & $4(2)$ & $4(2)$ & $5(3)$ \\
\hline \multicolumn{4}{|l|}{ Baseline SJC-44 } \\
\hline Mean (standard deviation) & $17.54(13.01)$ & $16.1(11.80)$ & $23.19(15.72)$ \\
\hline Median (inter-quartile range) & $14(19)$ & $13(18)$ & $22(25)$ \\
\hline \multicolumn{4}{|l|}{ Baseline RAI } \\
\hline Mean (standard deviation) & $12.63(11.11)$ & $11.64(10.45)$ & $16.48(12.69)$ \\
\hline Median (inter-quartile range) & $10(12)$ & $9(11)$ & $14(18)$ \\
\hline \multicolumn{4}{|l|}{ Baseline pain VAS } \\
\hline Mean (standard deviation) & $43.97(26.37)$ & $43.61(26.11)$ & $45.31(27.30)$ \\
\hline Median (inter-quartile range) & $45(40)$ & $45(38)$ & $45(43)$ \\
\hline \multicolumn{4}{|l|}{ Baseline ESR } \\
\hline Mean (standard deviation) & $42.17(28.79)$ & $42.53(28.88)$ & $40.79(28.42)$ \\
\hline Median (inter-quartile range) & $37(44)$ & $38(44)$ & $34(39)$ \\
\hline \multicolumn{4}{|l|}{ Baseline HAQ } \\
\hline Mean (standard deviation) & $1.15(0.77)$ & $1.16(0.77)$ & $1.1(0.77)$ \\
\hline Median (inter-quartile range) & $1(2)$ & $1(2)$ & $1(2)$ \\
\hline Seropositivity, $n(\%)$ & $914(62.39 \%)$ & $744(63.75 \%)$ & $170(57.05 \%)$ \\
\hline
\end{tabular}

$D A S$ Disease Activity Score, SJC swollen joint count, RAI Ritchie Articular Index, VAS Visual Analogue Scale, ESR erythrocyte sedimentation rate, $H A Q$ Health Assessment Questionnaire

\section{Regression imputation}

The fixed-effect estimates from the mixed-effects linear regressions for the univariable and multivariable regression imputations are shown in Table 2. The effect estimates give the following imputation formula for the univariate regression of DAS28 on DAS:

DAS28 $=($ DAS $\times 0.736)+1.247$.

The effect estimates from the multivariable regression of DAS28 on the square-root 44-SJC and RAI, the log ESR, pain VAS, age and sex are as follows:

$$
\begin{aligned}
\text { DAS28 }= & (\operatorname{sqrt}(\text { SJC44 }) \times 0.300)+(\operatorname{sqrt}(\mathrm{RAI}) \times 0.175) \\
& +(\log (\mathrm{ESR}) \times 0.739)+(\mathrm{PGA} \times 0.016) \\
& +(\text { female } \times-0.027)+(\operatorname{age} \times-0.001) \\
& +-0.086 .
\end{aligned}
$$

The estimated pseudo $R^{2}$ for the univariable regression and multivariable regression model was 0.73 and 0.91 , respectively, indicating high predictive accuracy. This was further supported by the results of the cross-fold validations (shown in supplementary material 1) which indicate similar pseudo
Table 2 Regression output from univariable and multivariable regression models

\begin{tabular}{lll}
\hline & Univariable imputation & $\begin{array}{l}\text { Multivariable imputa- } \\
\text { tion }\end{array}$ \\
\hline Observations & 1470 & 1470 \\
$N$ & 298 & 298 \\
DAS (95\% CI) & $0.736(0.71,0.76)$ & \\
SJC44 (95\% CI) & & $0.3(0.27,0.33)$ \\
RAI (95\% CI) & & $0.175(0.14,0.21)$ \\
ESR (95\% CI) & & $0.739(0.71,0.77)$ \\
PGA (95\% CI) & & $0.016(0.01,0.02)$ \\
Sex (95\% CI) & & $-0.027(-0.11,0.06)$ \\
Age (95\% CI) & & $-0.001(-0.00,0.00)$ \\
Constant (95\% CI) & $1.247(1.13,1.37)$ & $-0.086(-0.31,0.14)$ \\
Psuedo $R^{2}$ & 0.73 & 0.91 \\
\hline
\end{tabular}

$D A S$ Disease Activity Score, SJC swollen joint count, RAI Ritchie Articular Index, ESR erythrocyte sedimentation rate, $P G A$ patient global assessment

$R^{2}$ across the models for the univariable and multivariable regression: 0.72 and 0.91 , respectively. 
Fig. 1 a-c Bland and Altman plots testing the agreement between a DAS28 score and the van Gestel transformation, $\mathbf{b}$ DAS28 score and the univariable method, and $\mathbf{c}$ DAS28 score and the multivariable method (a) DAS28 vs. van Gestel

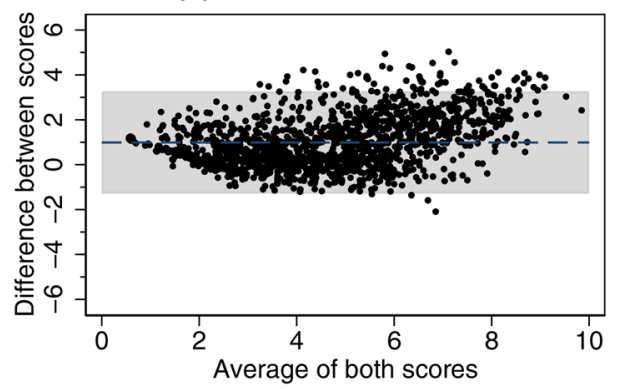

(b) DAS28 vs. Univariable

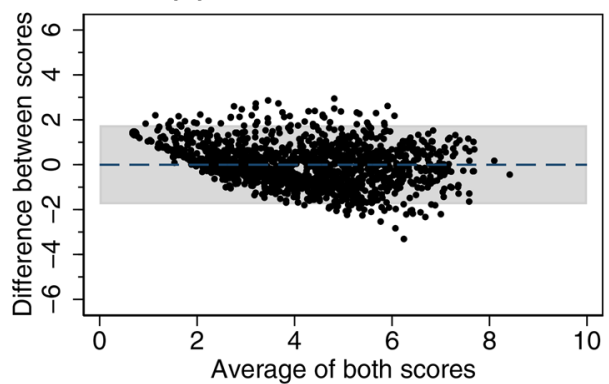

(c) DAS28 vs. Multivariable

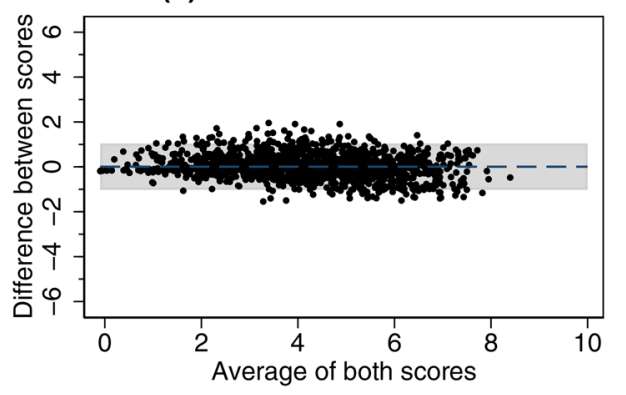

Bland and Altman plots (Fig. 1a-c) were used to investigate the level of agreement between the recorded DAS-28 scores and each of the three imputation methods. The largest bias was found for the van Gestel formula at 0.99 , along with the widest limits of agreement (LoA) at -1.29 to 3.27. The univariable regression imputation exhibited no bias (mean difference of -0.00 ), with slightly narrower LoA at $-1.76-1.76$, whilst the multivariable regression imputation also exhibited no bias (mean difference of 0.01 ), along with the narrowest LoA at -1.02 to 1.04. The plots highlight how for both the van Gestel and univariable regression method, there is a tendency for the lower scores to be overestimated.
Fig. 2 a-c Scatter concordance plots between a DAS28 score and the van Gestel transformation, b DAS28 score and the univariable method, and $\mathbf{c}$ DAS28 score and the multivariable method. The light grey line indicates the linear fit, and the dark grey line indicated the best fitting non-linear line (fractional polynomial)

\section{Concordance Plots}

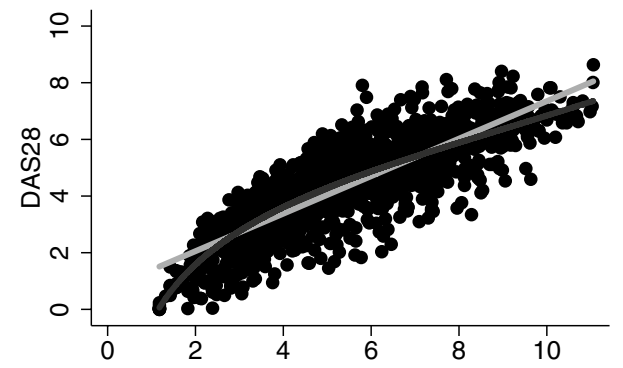

(a) DAS28(Van Gestel)

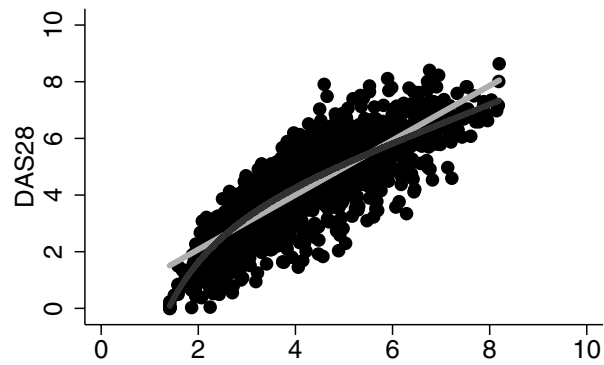

(b) DAS28(Univariable)

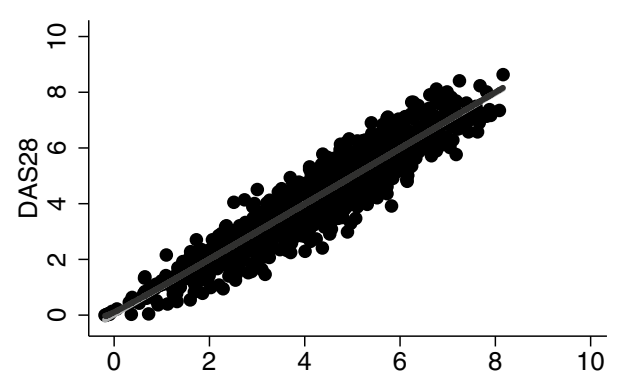

(c) DAS28(Multivariable) 
In fact, for both methods the minimum imputed DAS28 score observed is approximately one. Scatter plots showing concordance between each imputation method and the DAS28 score are shown in Fig. 2a-c. The red line is the reduced major axis of the data, which is equivalent to the slope of the correlation between the variables. The blue line shows the best fitting (non-linear) fractional polynomial line. This also highlights how both the van Gestel and univariable method overestimate true DAS28 scores at the lower and higher end of the scale, whereas the multivariable method indicates much closer agreement across the whole range of the score.

The Pearson correlation between the DAS28 and van Gestel formula was 0.85 , for the univariable regression was 0.85 and for the multivariable regression was 0.95 . The ICC for the transformation formula was the lowest at 0.72 (95\% confidence interval $0.69-0.74)$, followed by the regression imputation using only the DAS at 0.84 (95\% CI 0.83-0.86), and finally the highest ICC was observed for the multiple regression imputation with the component scores at 0.95 (95\% CI 0.95-0.96).

When categorised into remission, LDA, MDA and HDA, the recorded DAS2 8 scores indicated $22 \%$ of scores in remission, $12 \%$ of scores in LDA, $38 \%$ of scores in MDA and $28 \%$ of scores in HDA. The van Gestel formula saw an increased proportion of scores recorded as HDA at $45 \%$, with lower proportions in the other groups. Conversely, both the univariable imputation and the multivariable imputation indicated slight underestimation in the remission and HDA categories, and slight overestimation in the LDA and MDA categories.

The weighted Kappa agreement analysis of the DAS28 categories indicated the highest level of agreement between the multivariable imputation at $97.6 \%$ and a Kappa statistic of 0.91. Univariable imputation using only the DAS score indicated an agreement of $95.2 \%$, with a Kappa statistic of
0.81, whilst the transformation formula method indicated the lowest agreement at $93.6 \%$ and a Kappa statistic of 0.77 .

\section{DAS imputations over time}

The overall mean of the DAS28 over the first 5 years was 4.10 (SD 1.7). This compared to an overall mean of 5.09 (SD 2.19) using the van Gestel formula, 4.10 (SD 1.51) using univariable regression imputation of DAS and 4.11 (SD 1.63) using the multivariable regression imputation with the separate DAS components. While the mean scores were similar for the univariable and multivariable regression scores, the variability in the DAS28 was better approximated by the multivariable imputation model. The mean and standard deviations for each method over 5 years follow-up is given in Table 3. Figure 3 indicates the mean scores, along with the $95 \%$ confidence interval of the DAS28, the van Gestel formula, the univariable imputation and the multivariable imputation.

\section{Discussion}

Using a sub-sample of early RA patients from a longitudinal cohort, this study found that multivariable regression imputation of the DAS28 using the separate components of the original DAS measure resulted in the highest level of agreement when compared with recorded DAS28 scores. Furthermore, using the formula provided by van Gestel et al. [15], resulted in the lowest levels of agreement in this dataset. Whilst univariable regression imputation using only the total DAS score provided an improvement over the formula provided by van Gestel et al. [15], it showed similar levels of variability in the agreement when compared to the van Gestel transformation formula, across the spectrum of low to high scores.

Table 3 Mean (SD) of the recorded DAS28 score, along with the transformed DAS28 score using the van Gestel formula, univariable imputation and multivariable imputation

\begin{tabular}{|c|c|c|c|c|c|c|c|}
\hline & Total & Baseline & 1 year & 2 years & 3 years & 4 years & 5 years \\
\hline & $N=1499$ & $N=295$ & $N=237$ & $N=257$ & $N=243$ & $N=237$ & $N=230$ \\
\hline \multicolumn{8}{|l|}{ DAS28 } \\
\hline Mean (standard deviation) & $4.1(1.70)$ & $5.06(1.45)$ & $3.79(1.53)$ & $3.73(1.57)$ & $3.86(1.77)$ & $3.93(1.79)$ & $4.04(1.72)$ \\
\hline \multicolumn{8}{|l|}{ DAS28 van Gestel formula } \\
\hline Mean (standard deviation) & $5.09(2.19)$ & $6.14(2.05)$ & $4.69(1.96)$ & $4.63(2.11)$ & $4.89(2.21)$ & $4.94(2.23)$ & $5.01(2.22)$ \\
\hline \multicolumn{8}{|l|}{ DAS28 univariable imputation } \\
\hline Mean (standard deviation) & $4.1(1.51)$ & $4.82(1.41)$ & $3.82(1.35)$ & $3.78(1.45)$ & $3.96(1.52)$ & $3.99(1.53)$ & $4.04(1.53)$ \\
\hline \multicolumn{8}{|c|}{ DAS28 multivariable imputation } \\
\hline Mean (standard deviation) & $4.11(1.63)$ & $5.03(1.34)$ & $3.84(1.49)$ & $3.78(1.58)$ & $3.89(1.71)$ & $3.89(1.67)$ & $3.98(1.64)$ \\
\hline
\end{tabular}

$D A S$ Disease Activity Score 
Fig. 3 Mean and 95\% Confidence Intervals over the first 5 -years of the van Gestel transformation, univariable method and multivariable method, along with the recorded Disease Activity Score (DAS) 28

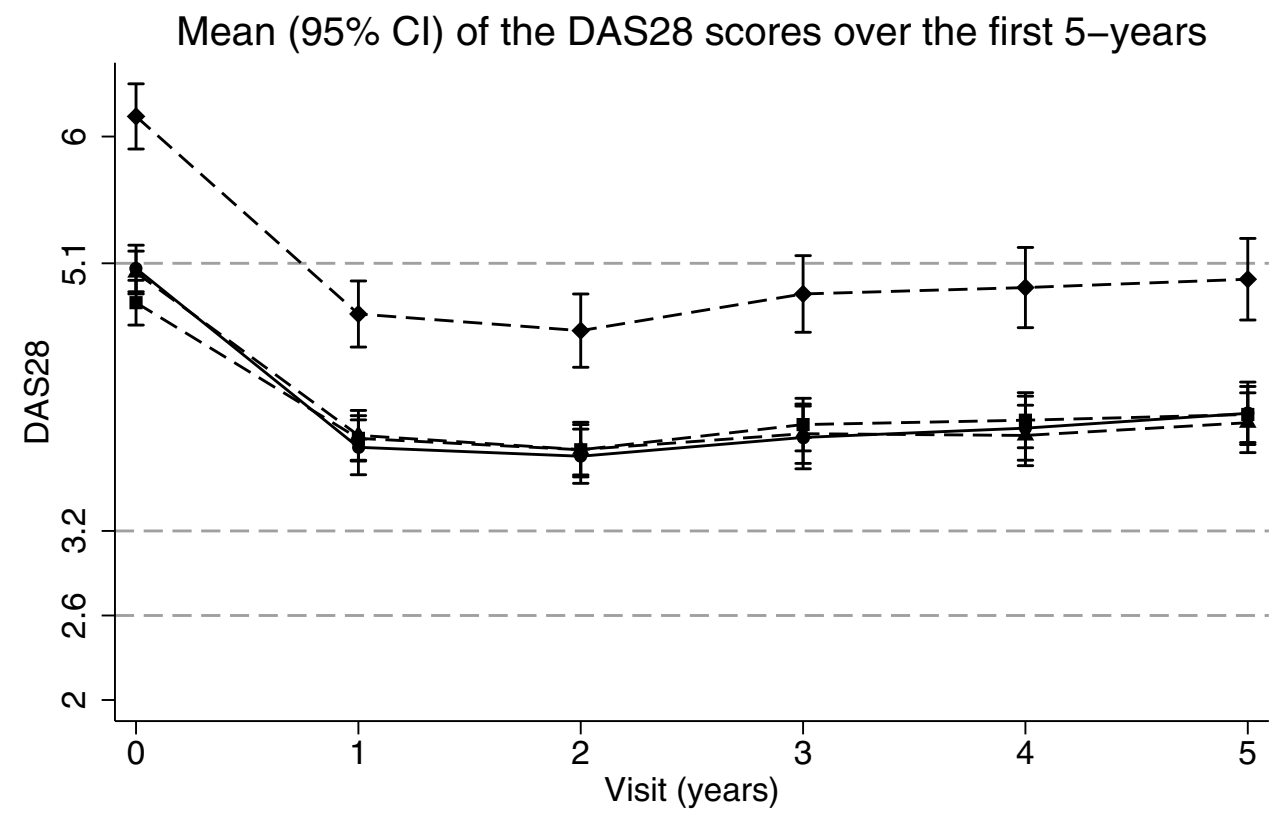

DAS28
The relatively large improvement in the mean difference between the univariable regression imputation using the total DAS in this analysis, compared to the formula by van Gestel et al. [15] suggests that using regression imputation formulae from different datasets may lead to some bias in the calculation of the transformed scores. Over the course of the first 5 years, the DAS28 using this formula was consistently more than one unit greater than the true DAS28 score, which resulted in much higher proportion of patients categorised into the HDA group. It is unclear why the univariable regression imputation devised using the ERAS data differed quite so significantly from the formula by van Gestel et al. [15], but it may be in part due to fundamental differences between the patient populations, such as differences between Dutch and UK patients, disease duration or disease severity that have impacted on the correlation between the two variables. Thus, ERAS is a cohort of patients with newly diagnosed RA where the components of the DAS will be less influenced by damage as would become apparent in patients with more longstanding disease. Unfortunately, the details of the derivation of the van Gestel formula are not clear, making it impossible to interrogate fully.

The analyses demonstrated that performing multivariable regression imputation using the separate components of DAS within the same dataset is the best approach for transforming DAS to DAS28. This is useful in situations where the DAS has been recorded but the DAS28 is preferred. Such instances include longitudinal studies considering secular trends over time $[11,14]$ and meta-analyses $[22,23]$. In such instances, where the DAS components are known, the authors recommend the use of the multivariable regression imputation formula outlined in this analysis in datasets of early RA, as this demonstrated greater agreement when compared to the van Gestel formula or the use of the univariable regression imputation formula.

Other forms of imputation, such as multiple imputation using chained equations (MICE) are also available, that have been shown to be less biased and more robust in imputing missing data [17]. Sensitivity analysis using a MICE approach was conducted (results not shown), however, it was not found to have worse levels of agreement compared to the multivariable regression imputation method outlined. This is to be expected since MICE treats the DAS28 score as unobserved, which introduces Monte Carlo error into the estimates. Given the additional steps involved in the calculation and use of MICE and multiply imputed data, the authors recommend that it should only be used in cases where individual components of the DAS28 are missing, to allow for more complete calculation of the DAS28 measure. In such cases, it is important that a measure of uncertainty around the imputation estimates is also given. However, for instances where only the original DAS is measured, the multivariable regression imputation method and formula outlined can be used.

Examination of demographic and baseline clinical variables for the sub-sample of patients with both DAS and DAS28 data indicated that, on average, these patients had higher disease activity at presentation. This could therefore indicate that the imputations were based on a subgroup of patients with more severe forms of RA. Interestingly, incidence of seropositivity and levels of ESR were lower than the rest of the cohort, suggesting that higher 
DAS may have been influenced more by TJC and pain VAS, the so-called subjective markers, rather than the more objective markers of inflammation [23, 24]. Nevertheless, it does suggest that these patients from which the multivariable regression imputation was derived, represent a marginally different sub-type of RA at presentation. Furthermore, the use of the pain VAS rather than the PGA VAS score for the majority of the DAS28 scores may have influenced the results. However, previous research has demonstrated that different measures of global health can be used interchangeably without significant impact on the DAS measure [16]. This, along with the very high agreement between the pain VAS and PGA VAS suggests that any bias is likely to be low from adopting this method. Moreover, this study represents the first analysis to investigate the use of different DAS scores in RA using one of the largest datasets of early RA patients, with long-term follow-up.

In summary, the analysis presented demonstrates the advantage of using multivariable regression imputation in situations where older data using DAS are to be converted to DAS28, to facilitate aggregation of data. While the best method is to use estimates from a regression model derived from the data itself, the paper provides a formula to transform data based on the separate components of the DAS, rather than just the overall DAS itself, in instances where data-specific parameters are not available. This formula provides an improved and statistically more reliable method for converting DAS to DAS28 for longterm analysis where research necessitates the combination of historical and contemporary data in RA.

Acknowledgements This paper has been conducted using data generated from the Early Rheumatoid Arthritis Study (ERAS). We are indebted to all the nurses and rheumatologists for their participation and contribution. We offer special thanks to Dr Nigel Cox and his staff for collecting the additional data needed for the analysis presented in this paper.

Author contributions LC undertook the analysis and prepared the manuscript. SN supported with the analysis and helped prepare the manuscript. Dr Nikiphorou helped prepare the manuscript. PK, DAW, JD and AY helped collect the data used for the analysis and helped prepare the manuscript.

Funding The work conducted in this study was funded by MQ/ARUK project grant (MQ16IP18).

\section{Compliance with ethical standards}

Conflict of interest Dr Carpenter declares that he has no conflict of interest. Dr Norton declares that he has no conflict of interest. Dr Nikiphorou declares that she has no conflict of interest. Dr Kiely declares that he has no conflict of interest. Dr Walsh reports grants and personal fees from Pfizer Ltd, personal fees from GSK Consumer Healthcare. Dr Dixey declares that he has no conflict of interest. Dr Young declares that he has no conflict of interest.
Ethical standards All procedures performed in studies involving human participants were in accordance with the ethical standards of the institutional and/or national research committee and with the 1964 Helsinki declaration and its later amendments or comparable ethical standards.

Informed consent Informed consent was obtained from all individual participants included in the study.

Open Access This article is distributed under the terms of the Creative Commons Attribution 4.0 International License (http://creativeco mmons.org/licenses/by/4.0/), which permits unrestricted use, distribution, and reproduction in any medium, provided you give appropriate credit to the original author(s) and the source, provide a link to the Creative Commons license, and indicate if changes were made.

\section{References}

1. Van Der Heijde D, van't Hof MA, van Riel PL et al (1990) Judging disease activity in clinical practice in rheumatoid arthritis: first step in the development of a disease activity score. Ann Rheum Dis 49(11):916-920. https://doi.org/10.1136/ ard.49.11.916

2. Smolen JS, Breedveld FC, Burmester GR et al (2016) Treating rheumatoid arthritis to target: 2014 update of the recommendations of an international task force. Ann Rheum Dis 75(1):3-15. https://doi.org/10.1136/annrheumdis-2015-207524

3. Scott DL (2012) Biologics-based therapy for the treatment of rheumatoid arthritis. Clin Pharmacol Ther 91(1):30-43

4. Prevoo MLL, Van'T Hof MA, Kuper HH, Van Leeuwen MA, Van De Putte LBA, Van Riel PLCM (1995) Modified disease activity scores that include twenty-eight-joint counts development and validation in a prospective longitudinal study of patients with rheumatoid arthritis. Arthritis Rheum 38(1):4448. https://doi.org/10.1002/art.1780380107

5. Landewé R, van der Heijde D, van der Linden S, Boers M (2006) Twenty-eight-joint counts invalidate the DAS28 remission definition owing to the omission of the lower extremity joints: a comparison with the original DAS remission. Ann Rheum Dis 65(5):637-641. https://doi.org/10.1136/ard.2005.039859

6. Van Der Heijde, D., \& Jacobs, J. W. (1998). The original "DAS" and the "DAS28" are not interchangeable: comment on the articles by Prevoo et al. Arthritis Rheum 41(5):942-945. https:// doi.org/10.1002/1529-0131(199805)41:5\%3C942::AID-ART26 \%3E3.0.CO;2-B

7. Boers M, Tugwell P, Felson DT, van Riel PL et al (1994) World Health Organization and International League of Associations for Rheumatology core endpoints for symptom modifying antirheumatic drugs in rheumatoid arthritis clinical trials. J Rheumatol Suppl 41:86-89

8. Symmons DP, Barrett EM, Bankhead CR, Scott DG, Silman AJ (1994) The incidence of rheumatoid arthritis in the United Kingdom: results from the Norfolk Arthritis Register. Br J Rheumatol 33(8):735-739

9. Twigg S, Hensor EMA, Emery P, Tennant A, Morgan AW, Yorkshire Early Arthritis Register Consortium (2017) Patientreported outcomes as predictors of change in disease activity and disability in early rheumatoid arthritis: results from the yorkshire early arthritis register. J Rheumatol 44(9):1331-1340. https://doi.org/10.3899/jrheum.161214

10. Carpenter L, Nikiphorou E, Young A (2013) Importance of registries in informing clinical practice for arthritis. Clin Pract. https://doi.org/10.2217/cpr.13.70 
11. Nikiphorou E, Norton S, Young A, Carpenter L, Dixey J, Walsh DA, Kiely P (2016) Association between rheumatoid arthritis disease activity, progression of functional limitation and long-term risk of orthopaedic surgery: combined analysis of two prospective cohorts supports EULAR treat to target DAS thresholds. Ann Rheum Dis. https://doi.org/10.1136/annrheumdis-2015-208669

12. Kroot, E. J., de Jong, B. A., van Leeuwen, M. A., et al. (2000). The prognostic value of anti-cyclic citrullinated peptide antibody in patients with recent-onset rheumatoid arthritis. Arthritis Rheum 43(8):1831-1835. https://doi.org/10.1002/1529. 0131(200008)43:8\%3C1831::AID-ANR19\%3E3.0.CO;2-6

13. Fransen J, Creemers MCW, Van Riel PLCM (2004) Remission in rheumatoid arthritis: agreement of the disease activity score (DAS28) with the ARA preliminary remission criteria. Rheumatology 43(10):1252-1255. https://doi.org/10.1093/rheumatolo gy/keh297

14. Carpenter L, Norton S, Nikiphorou E et al (2017) Reductions in radiographic progression in early RA over 25 -years: changing contribution from RF in 2 multi-centre UK inception cohorts. Arthritis Care Res. https://doi.org/10.1002/acr.23217

15. Van Gestel, A. M., Haagsma, C. J., \& van Riel, P. L. (1998). Validation of rheumatoid arthritis improvement criteria that include simplified joint counts. Arthritis Rheum 41(10):1845-1850. https ://doi.org/10.1002/1529-0131(199810)41:10\%3C1845::aid-art17 \%3E3.0.co;2-k

16. Khan NA, Spencer HJ, Abda EA et al (2012) Patient's global assessment of disease activity and patient's assessment of general health for rheumatoid arthritis activity assessment: are they equivalent? Ann Rheum Dis 71(12):1942-1949. https://doi. org/10.1136/annrheumdis-2011-201142
17. Sterne J, White IR, Carlin JB et al (2009) Multiple imputation for missing data in epidemiological and clinical research: potential and pitfalls. BMJ (Clin res) 338:b2393. https://doi.org/10.1136/ bmj.b2393

18. Radovits BJ, Fransen J, van Riel PLCM, Laan RFJM (2008) Influence of age and gender on the 28-joint Disease Activity Score (DAS28) in rheumatoid arthritis. Ann Rheum Dis 67(8):11271131. https://doi.org/10.1136/ard.2007.079913

19. Altman DG, Bland JM (1983) Measurement in medicine: the analysis of method comparison studies. Statistician 32:307-317

20. Fransen J, Van Riel P (2005) The Disease Activity Score and the EULAR response criteria. Clin Exp Rheumatol 23(Supp 39):S93S99. https://doi.org/10.1016/j.rdc.2009.10.001

21. McHugh ML (2012) Interrater reliability: the kappa statistic. Biochem Med 22(3):276-282

22. Carpenter L, Nikiphorou E, Sharpe R et al (2016) Have radiographic progression rates in early rheumatoid arthritis changed? A systematic review and meta-analysis of long-term cohorts. Rheumatology. https://doi.org/10.1093/rheumatology/kew004

23. Navarro-Compan V, Gherghe AM, Smolen JS, Aletaha D, Landewe R, Van Der Heijde D (2015) Relationship between disease activity indices and their individual components and radiographic progression in RA: a systematic literature review. Rheumatology (United Kingdom). https://doi.org/10.1093/rheumatolo gy/keu413

24. Walsh DA, McWilliams DF (2014) Mechanisms, impact and management of pain in rheumatoid arthritis. Nat Rev Rheumatol 10(10):581-592. https://doi.org/10.1038/nrrheum.2014.64 